\title{
Adhesive-hydrogel composite developed to repair damaged cartilage
}

Focal cartilage lesions rarely heal and are associated with joint degeneration. Cartilage defects are currently treated by microfracture surgery: small holes are created in the subchondral bone underneath a cartilage defect creating a blood clot, which enables the cartilage lesion to be filled. A photoreactive adhesive-hydrogel composite has been developed which, when used in microfracture, improved tissue fill and reduced pain in a pilot clinical study published in Science Translational Medicine.

Poly(ethylene glycol) hydrogels do not adhere well to tissue, so the investigators used a chondroitin sulfate adhesive to immobilize the hydrogel in the lesion. The hydrogel was introduced as a solution so it could fill irregularly shaped holes; on exposure to light the gel polymerizes to form a solid implant. The composite was tested in vitro, in animal models and then in patients with a single focal cartilage defect $2-4 \mathrm{~cm}^{2}$ in size on the medial femoral condyle. Lesion fill was evaluated by MRI 3 and 6 months after the procedure. Repair tissue filled $86 \%$ of the lesion in patients treated with the hydrogel $(n=15)$, and filled $64 \%$ in patients treated with microfracture alone $(n=3)$. Patients treated with the hydrogel reported less severe and less frequent pain than those treated with microfracture alone. However, no differences were seen between the groups' median IKDC score, a measure of both pain and disability. No major adverse events were observed in the 6 months after microfracture surgery.

Nonadhesive cells such as chondrocytes survive in hydrogels, while adhesive cells such as osteoblasts do not. The chondrocyte progenitor cells needed for tissue repair come from the bone marrow, are released into the lesion by the microfractures and
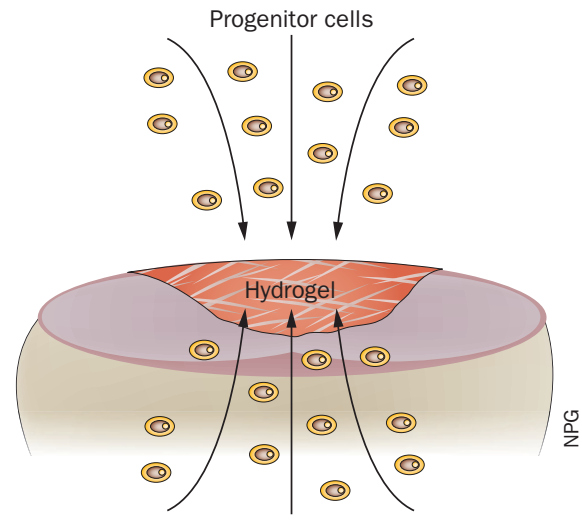

then become embedded in the hydrogel. "We're using the body's native capacity for repair and endogenous cells, and using a biomaterial to concentrate and guide the process," says lead researcher Jennifer Elisseeff. She plans to continue to modify the hydrogel, aiming to reduce inflammation and improve lubrication.

Megan Cully

Original article Sharma, B. et al. Human cartilage repai with a photoreactive adhesive-hydrogel composite. Sci. Transl. Med. doi:10.1126/scitransImed.3004838 